\title{
Changes in the immune system - the key to diagnostics and therapy of patients with non-alcoholic fatty liver disease
}

\author{
MARCIN KOSMALSKI I , ŁUKASZ MOKROS ${ }^{1}$, PIOTR KUNA², ANDRZEJ WITUSIK ${ }^{3}$, \\ TADEUSZ PIETRAS ${ }^{l}$
}

'Department of Clinical Pharmacology, Medical University of Lodz, Poland

${ }^{2}$ Department of Internal Medicine, Asthma and Allergy, Medical University of Lodz, Poland

${ }^{3}$ Department of Psychology, Piotrków Trybunalski Branch, Jan Kochanowski University in Kielce, Poland

\begin{abstract}
Non-alcoholic fatty liver disease (NAFLD) is one of the most common pathologies of that organ. The development of the disease involves a variety of mechanisms, including insulin resistance, oxidative stress, endoplasmic reticulum stress, endotoxins from the intestinal flora and genetic predispositions. Additionally, clinical data suggest that the presence of NAFLD is associated with excessive activation of the immune system. For practical purposes, attention should be paid to the moment when the subjects predisposed to NAFLD develop inflammatory infiltration and signs of fibrosis in the liver (non-alcoholic steatohepatitis - NASH). Their presence is an important risk factor for hepatic cirrhosis, hepatic failure, and hepatocellular carcinoma, as well as for the occurrence of cardiovascular events. Regardless of the diagnostic methods used, including laboratory tests and imaging, liver biopsy remains the gold standard to identify and differentiate patients with NAFLD and NASH. The search for other, safer, cheaper and more readily available diagnostic tests is still being continued. Attention has been drawn to the usefulness of markers of immune status of the organism, not only for the diagnosis of NASH, but also for the identification of NAFLD patients at risk of disease progression. Despite the effectiveness of medication, no recommendations have been established for pharmacotherapy of NAFLD. Data indicate the primary need for non-pharmacological interventions to reduce body weight. However, there is evidence of the applicability of certain drugs and dietary supplements, which, by their effect on the immune system, inhibit its excessive activity, thus preventing the progression of NAFLD to NASH.
\end{abstract}

Key words: therapy, diagnosis, immune system, non-alcoholic fatty liver disease.

(Centr Eur Immunol 2018; 43 (2): 231-239)

\section{Introduction}

Non-alcoholic fatty liver disease (NAFLD) is defined as an excessive accumulation of fat in the liver in individuals not consuming alcohol, or consuming it in small amounts, i.e., according to the most common considerations, $\leq 20$ grams for women and for men $\leq 30$ grams per day $[1,2]$. An additional criterion for the diagnosis of this pathology is the need to eliminate any other, secondary causes of fatty infiltration of the liver, which include, inter alia, HCV infection, the use of glucocorticoids, celiac disease, Wilson disease, familial hypobetalipoproteinemia and presence of specific autoantibodies (autoimmune hepatitis - AIH) such as antinuclear antibodies (ANAs) and/or antismooth muscle antibodies (ASMAs) for AIH1 and antiliver kidney microsome 1 (LKM 1) for AIH2 [3, 4]. Also, NAFLD is assumed if $5 \%$ or more of the hepatocytes in a liver biopsy have macrovesicular steatosis, or the proton magnetic resonance spectroscopy-derived liver fat content is $5.56 \%$ or higher [5]. NAFLD is not a single disease entity. It covers a broad spectrum of irregularities, ranging from simple fatty liver (NAFL), through the development of inflammation and fibrosis (NASH), to full-blown cirrhosis of the organ, increasing over 2.5 -fold the risk of hepatocellular carcinoma (HCC) [6]. It has been demonstrated that in some patients the disease may be progressive in character. It is estimated that within three years $23-39 \%$ of NAFL patients will develop NASH, among which $10-25 \%$ will progress to full-blown cirrhosis $[7,8]$. Therefore, particular attention should be paid to the moment when lobular inflammatory infiltration leading to damage to hepatocytes (ballooning degeneration), with or without peri-sinusoidal fibrosis typical of NASH - appears in patients with NAFLD [9].

Correspondence: Marcin Kosmalski, PhD, Department of Clinical Pharmacology, Medical University of Lodz, 22 Kopcińskiego St., 91-153 Lodz, Poland, e-mail: marcin.kosmalski@umed.lodz.pl Submitted: 19.02.2017; Accepted: 12.06.2017 
Cirrhosis and liver cancer are not the only pathologies associated with the risk of death in these patients. It has been proven that the main factors responsible for high, up to $34 \%$, mortality are cardiovascular incidents [10].

The data to assess the incidence of this disease are divergent and estimated at 4-46\%, depending on the diagnostic method used [11]. NAFLD is commonly considered to be a component of metabolic syndrome (MS), due to the frequent occurrence of concomitant insulin resistance (IR), obesity, lipid metabolism disorders, as well as type 2 diabetes mellitus (T2DM). It must be emphasized, however, that this coincidence does not apply to all patients. It is estimated that NAFLD occurs in about $30 \%$ of obese men and $40 \%$ of obese women and in $70 \%$ of patients with T2DM, and the diagnostic criteria for MS are met only by $33 \%$ of patients [12-14].

The development of fatty liver disease and its consequences are not completely understood and explained. There are several theories associated with the presence of this pathology. The most popular of them is the theory of two impacts, the first impact being the presence of fatty infiltration in the liver due to accumulation of triglycerides in hepatocytes, leading to IR. The excess fat, in the second impact, can trigger mechanisms leading to the development of inflammation, fibrosis, and necrotic cell death. Such mechanisms include oxidative stress, endoplasmic reticulum stress, cytokines produced by several cell types and endotoxins from the intestinal flora [15]. In addition, endocrine system disorders and genetic predispositions contribute to the development of this pathology [16]. For example, insulin-like growth factor 2 (IGF-2) is expressed excessively in the liver when the immune system is over-activated, compared to healthy controls. It is suggested that the gene for IGF-2 may be NASH-specific. In addition, patients with NAFLD had significantly lower insulin-like growth factor 1 (IGF-1) activity. It is believed that this is related to a decrease in insulin sensitivity, and lipid oxidation, probably independently of total or visceral adiposity $[17,18]$.

In order to diagnose excess fat in the liver, several methods, differing in sensitivity, specificity, availability and cost of implementation, are used. Among them, routine laboratory tests [alanine aminotransferase (ALT), aspartate aminotransferase (AST), gamma glutamyltranspeptidase (GGTP), a2-macroglobulin, apolipoprotein-1, bilirubin, cholesterol, triglycerides (TG) and insulin levels], imaging studies (ultrasound (USG), computed tomography (CT), magnetic resonance imaging (MRI) and elastography) as well as scoring systems based on the results of the above tests, the patient's age and gender, comorbidities and anthropometric measurements should be mentioned [19]. Despite the fact that ultrasound examination is the main diagnostic tool, it does not enable one to assess the presence of an inflammatory condition, fibrosis and degeneration of hepatocytes in the liver [20]. The gold standard diagnostic tool for inflammatory infiltration, fibrosis and cellular damage is liver biopsy.
Nevertheless, it is performed infrequently in daily clinical practice due to its invasive nature, high costs, rare availability and, above all, the risk of complications including those with a fatal outcome, [21, 22]. Additionally, obtaining a biopsy appropriate for histopathological evaluation is difficult [23]. For this reason, the search for other, non-invasive and inexpensive methods that would not only make it possible to diagnose patients with NAFLD with the highest sensitivity and specificity, but also enable one to assess the degree of damage to the organ and to identify people at risk of disease progression, is still ongoing. Therefore, the usefulness of investigations assessing the immune system, which could be incorporated into routine diagnosis, has been suggested for this purpose [24, 25].

The therapeutic options are based mainly on the need to reduce body weight. To date, no algorithm of pharmacological management has been developed due to lack of sufficient evidence of the effectiveness of the investigational drugs and dietary supplements, including hypoglycemic and hypolipidemic agents, weight loss medications, angiotensin II receptor antagonists, vitamin E, ursodeoxycholic acid, obeticholic acid, emricasan, and $n-3$ polyunsaturated fatty acids $[20,26]$. There are, however, indications of potentially effective application of the drugs interfering with the immune system in patients with NASH [27].

Therefore, the purpose of this paper is to present the diagnostic opportunities associated with the immune system in patients with NAFLD, not yet diagnosed with NASH, as well as to assess the options of therapeutic intervention in the immune system in this group of patients.

\section{NAFLD and the immune system}

The liver, in addition to hepatocytes and cholangiocytes, contains lymphocytes, monocytes, Kupffer cells, NK, NKT, astrocytes dendritic and a large amount of other immigrant cells associated with the immune system. Additionally, it has been proven that the organ is the site where synthesis of various proteins such as acute-phase protein, complement, cytokines, chemokines, lipid messengers as well as reactive oxygen species (ROS) takes place [28, 29]. The main objective of inflammatory processes in the liver is not only to combat infections, and promote physiological processes, such as hemodynamic changes, permeability of the capillaries, migration of white blood cells to the tissues, secretion of inflammatory mediators, but also to promote apoptosis and necrotic demise of hepatocytes [30, 31]. The liver is a unique immune environment, which is exposed to a variety of immunogenic factors from the gastrointestinal tract, including nutrients and elements of Gram-negative bacterial wall structure. If any of these factors are present, the immune system of the liver is subjected to improper activation, leading to the development of an inflammatory condition, cell damage, fibrosis, organ dysfunction and carcinogenesis [32]. In the case of obesity, inflammation 
involves not only the liver, but also the adipose tissue, the pancreas and the muscles, which is associated with IR and metabolic dysfunction. A disequilibrium between the pro- and anti-inflammatory cytokines causes changes in response to insulin, $\beta$-oxidation, storage and transport of lipids, autophagy and nuclear receptor signaling, resulting in progression of the disease to NASH [33]. The immune system is closely related to the hormonal system. For example, glucocorticoids (GCs) are synthesized and secreted from the adrenal cortex and are involved in the regulation of carbohydrate and lipid metabolism, reproduction, and growth, as well as in the anti-inflammatory and immune response. GCs exert their effects through the glucocorticoid receptors (GC-GRs). Patients with NASH were shown to have increased hepatic expression of GC-GR [34]. Additionally, the enzyme which regenerates cortisol from cortisone (11 $\beta$-hydroxysteroid dehydrogenases type $1,11 \beta$-HSD1) is highly expressed in the liver. It has been shown to regulate hepatic glucose output and be linked to intrahepatic fat deposition and indirectly influence the proinflammatory state. It has been shown that inhibition of this enzyme results in significant reduction of liver-fat content and decrease in activity of ALT and GGTP, without significant changes in insulin sensitivity [35].

\section{The immune system and diagnostics of NAFLD}

\section{C-reactive protein, procalcitonin and leukocytes}

The assessment of leukocyte (WBC) count, concentration of C-reactive protein (CRP), and procalcitonin (PCT) are investigations often performed both in diagnostics and in monitoring pharmacotherapy of many diseases because they are readily available, reliable, and cheap. CRP and PCT belong to acute phase proteins [36]. CRP synthesis takes place mainly in the liver and is associated with the acute phase of inflammation [37]. PCT under normal conditions is secreted by the $\mathrm{C}$ cells of the thyroid gland, whereas in the case of a bacterial infection it is also synthesized ectopically by a variety of tissues and cells, including monocytes and macrophages [38, 39]. It has been proven that patients with NAFLD have higher concentrations of the highly sensitive form of CRP (high-sensitivity CRP hsCRP) and a larger number of leukocytes as compared with patients in whom the US scan of the liver does not reveal signs of fatty infiltration. It should be emphasized that these values are independent of other risk factors for NAFLD, such as waist circumference, presence of IR and T2DM, and TG levels. They have also been proven to correlate with the amount of fat in the liver [40]. Orunc et al., who compared 50 patients with histopathologically diagnosed NAFLD and NASH with the same number of subjects with normal ultrasound image of the liver, found no significant differences in the concentration of PCT. In contrast, they observed a difference in the case of CRP concentration. Patients in the study group had higher levels of this protein, but the presence of an inflammatory condition and fibrosis in histopathology did not significantly affect the concentration of this protein. It is noteworthy that three patients, who had only focal fatty infiltrations visualized on USG, demonstrated normal PCT and CRP concentrations [41]. Significantly higher hs-CRP values were also found in the case of coexistence of NAFLD and T2DM. It is suggested, however, that in this group of patients this protein is not an independent factor for the presence of excess fat in the liver [12, 42].

It has been proven that the presence of excess fat in the liver in US images in patients with NAFLD correlates positively with the values of WBC, which, by means of neutrophils and lymphocytes, play a key role in the body's immune defense [43, 44]. The neutrophil-to-lymphocyte ratio (NLR), which integrates the detrimental effects of neutrophilia (an indicator of inflammatory condition) and lymphopenia (an indicator of physiological stress), has emerged as a useful prognostic marker of subclinical systemic inflammation [45, 46]. Kara et al. demonstrated that patients with NAFLD, in whom NASH was included on the basis of histopathological investigations, had significantly lower WBC and neutrophil counts. There were, however, no differences in the counts of lymphocytes and NLR [47]. A study by Abdel-Razik et al. demonstrated that patients with confirmed NASH had higher NLR as compared to patients with hepatic steatosis but without the presence of the common characteristics of NASH. It should be noted that NLR correlated positively with other immune markers, such as CRP, tumor necrosis factor- $\alpha$ (TNF- $\alpha$ ) and interleukin-6 (IL-6). The receiver-operating characteristic (ROC) curve analysis indicated that at a cut-off value of 2.05, the NLR has the highest specificity $(75.4 \%)$ and sensitivity $(73.2 \%)$ for identification of NASH patients with an area under the curve (AUC) of 0.773 (95\% CI: $0.571-0.789, p<0.001$ ), with positive predictive value (PPV) and negative predictive value (NPV) for NLR of 78.6 and $69.2 \%$, respectively [48].

\section{Proinflammatory cytokines and other mediators of inflammation}

Proinflammatory cytokines constitute a heterogeneous group of molecules secreted by different cells, causing a variety of biological effects. They act as endogenous pyrogens, increase the synthesis of secondary mediators involved in the inflammatory process by macrophages and mesenchymal cells, stimulate the production of acute phase proteins and attract inflammatory cells [49]. It has been suggested that TNF- $\alpha$ and interleukin-1 (IL-1) are among the most important mediators of NAFLD development. In obese patients with NAFLD, TNF- $\alpha$ is produced by adipose tissue, causing peripheral impairment, insulin-depen- 
dent glucose metabolism and stimulating hormone sensitive lipase, thus causing an increase in the concentration of free fatty acids (FFA) and their inflow to the liver. In addition, the generators of this cytokine in the liver are Kupffer cells that produce TNF- $\alpha$ in response to bacterial toxins, and the process is mediated by toll-like receptors (TLR) [50, 51]. Elevated TNF- $\alpha$ in patients without signs of NAFLD evident on USG has been proven to be associated with a high risk of developing fatty liver within a 4-year follow-up period [52]. IL-1 constitutes a family of proinflammatory and membrane cytokines produced by macrophages, endothelial cells and fibroblasts. IL-1 $\alpha$ and IL-1 $\beta$ have been proven to play a significant role in the transformation of NAFLD to NASH [53]. Moreover, it has been suggested that IL- $1 \beta$ in a complex mechanism may promote the development of NAFLD in patients with IR [54]. Animal model studies suggest that the IL-1Ra receptor for IL-1 $\beta$ and IL-1 $\alpha$ may have a function protective against the development of fatty infiltration of the liver [55]. In the case of IL- $1 \alpha$, no changes in its concentration in patients with NAFLD have been confirmed [56].

Interleukin-6 (IL-6) plays the role of a proinflammatory cytokine, produced, among others, by adipocytes, hepatocytes and immune cells. Endotoxemia in obesity, resulting from small intestinal bacterial overgrowth, stimulates macrophages through TLR receptors to produce TNF- $\alpha$ that possibly upregulates IL- 6 production from adipocytes and macrophages infiltrated in adipose tissue. Polyzos et al. found no significant difference in the concentration of cytokines in the situation of emergence of the characteristics of fatty liver revealed by biopsy. They proved that patients with simple steatosis of the organ and with the presence of signs of NASH have similar values of this cytokine. The difference has been reported only in the case of a comparison of the group of patients without signs of fatty liver with NASH patients [56].

It has been shown that interleukin-15 (IL-15) acts as a growth factor for T-lymphocytes and affects many other cells of the immune system. Its main function is associated with improvement of CD8+ T cell survival, involvement in the production and maintenance of NK cells, NKT cells, $\gamma \delta$ of T cells and a subset of T cells in the intestines. It has also been proven to enhance the survival of DC and macrophages and to stimulate them to produce cytokines. This facilitates the development of a potent immune response. Its biological effect is mediated predominantly by Il-15: Il$15 \mathrm{R} \alpha$ complex. Cepero-Donates et al. demonstrated in an animal model of NAFLD that the lack of this interleukin reduces accumulation of fat in the liver, inflammation and the recruitment of immune cells. They also showed that Il-15 in the liver acts directly on hepatocytes, inducing the expression of chemokines, which may be responsible for the recruitment of immune cells [57].

Another cytokine that may be applicable in the diagnosis of NAFLD is interleukin-18 (IL-18), produced by macrophages, Kupffer cells and the endothelium. It induces production of chemokines, adhesion molecules and proinflammatory cytokines. IL-18 binding protein, an inhibitor that binds to the same receptor as IL-18, enhances its negative feedback mechanism enabling cell protection from accelerated proinflammatory activity such as NASH. Li Y et al. demonstrated that patients with NAFLD have significantly higher levels of this interleukin and the value of the IL-18/IL-8 binding protein index as compared with the group without excess fat in the liver [58]. The interleukin-17 axis is a large family of pro-inflammatory cytokines, including, among others, interleukin-17A (IL-17A) and interleukin-17F (IL-17F). As demonstrated in the animal model of NAFLD, IL-17A and IL-7F are produced in the liver by T CD4+ and CD8+ cells, and their signal is mediated by the IL-17RA receptor, whose expression is also increased in the case of fatty infiltration of the liver. This process initiates a cascade of events, including a change in the phenotype of macrophages in the proinflammatory direction [59]. Th17 cells produce IL17 (also referred to as IL-17A), IL-17F, IL-21 and IL-22. Tang et al. observed in an animal model that Th17 cells and IL-17 were associated with hepatic steatosis and the proinflammatory response in NAFLD and facilitated the transition from simple steatosis to NASH [60].

Interferon gamma-induced protein 10 (IP-10), regarded as a proinflammatory cytokine, regulates activation and recruitment of white blood cells, including lymphocytes, monocytes and natural killer cells, by binding to chemokine (C-X-C motif) receptor 3 (CXCR3). In addition, this substance is secreted by a variety of other cells including monocytes, neutrophils, endothelial cells, keratinocytes, fibroblasts, mesenchymal cells, dendritic cells, astrocytes, and hepatocytes. As observed by Chang et al. in a group of patients with histopathologically confirmed NAFLD, the presence of excess fat in the liver is associated with a significantly higher concentration of this interleukin, the value of which significantly increases in the case of NAFLD coexistence with diabetes. It should be noted that the level of IP10 correlated positively with other immunological markers such as CRP, monocyte chemoattractant protein-1 (MCP-1) and TNF- $\alpha$ [42].

Transforming growth factor beta (TGF- $\beta$ ) has three isomers (TGF- $\beta 1$, TGF- $\beta 2$, and TGF- $\beta 3$ ), which are secreted by a variety of cells, including Kupffer cells, astrocytes and hepatocytes. It has been proven that patients with elevated TGF- $\beta 3$ are at increased risk of the development of NAFLD in 4-year follow-up [61].

Vascular adhesion protein-1 (VAP-1) is a protein molecule presenting on the hepatic endothelial cells. Its role is to promote adhesion and migration of lymphocytes across the primary hepatic sinusoidal endothelium. Patients with histologically confirmed NAFLD have been demonstrated to have a higher concentration of this molecule, compared to 
sex-, age-, and metabolic phenotype-matched patients without signs of fatty liver disease [62].

\section{The immune system and therapy of NAFLD}

There are only a few research articles considering the effectiveness of therapy targeting NAFLD and its progression to NASH by affecting the immune system. Available data are summarized in Table 1.

The basis for the therapy of NAFLD is a reduction of body weight based on a suitable reducing diet and moderate exercise. Larson-Meyer et al. in a group of 46 obese patients applied a medication-free procedure based on the above recommendations. Based on imaging studies, such as MRI or CT scans, they found surprisingly that such a drug-free procedure is associated with a slight increase of hsCRP, elevation of IL-6 and insignificant changes in TNF- $\alpha$ concentration [63].

During a 6-month follow-up of patients using barnidipine or perindopril, it was found that only the calcium channel blocker significantly reduces the concentration of TNF- $\alpha$, IL-6, and hs-CRP both in monotherapy and in combination therapy with simvastatin. There were, however, no changes in the liver ultrasound image in the case of the two drugs used alone. A significantly greater opportunity to improve this image was provided by adding an inhibitor of HMG-CoA reductase to either of these drugs [64].

Beneficial immunomodulatory properties in the case of NAFLD have been demonstrated for fenofibrate in monotherapy as well as in combination therapy with pentoxi-

Table 1. Effectiveness of therapy targeting NAFLD and its progression to NASH by affecting the immune system

\begin{tabular}{|c|c|c|c|c|c|}
\hline Author & $\begin{array}{c}\text { Number } \\
\text { of patients }\end{array}$ & $\begin{array}{l}\text { Diagnostic } \\
\text { methods }\end{array}$ & Intervention & Duration & Results \\
\hline $\begin{array}{l}\text { Larson-Meyer } \\
\text { DE [63] }\end{array}$ & 46 & MRI, CT & $\begin{array}{c}\text { Caloric restriction and } \\
\text { increased structured exercise }\end{array}$ & 6 months & No change in TNF- $\alpha$, IL- 6 and hs-CRP \\
\hline Derosa G [64] & 149 & USG & $\begin{array}{l}\text { Perindopril } 5 \mathrm{mg} / \text { day or } \\
\text { barnidipine } 20 \mathrm{mg} / \text { day }\end{array}$ & 6 months & $\begin{array}{l}\text { Only barnidipine reduced TNF- } \alpha \text {, IL- } 6 \\
\text { and hs-CRP in monotherapy and after } \\
\text { simvastatin addition }\end{array}$ \\
\hline $\begin{array}{l}\text { El-Haggar MS } \\
{[65]}\end{array}$ & 90 & $\begin{array}{l}\text { USG and elevated } \\
\text { aminotransferase }\end{array}$ & $\begin{array}{c}\text { Fenofibrate } 300 \mathrm{mg} / \text { day or } \\
\text { fenofibrate } 300 \mathrm{mg} / \text { day plus } \\
\text { pentoxifylline } 1200 \mathrm{mg} / \text { day in } \\
\text { three divided doses }\end{array}$ & 24 weeks & $\begin{array}{l}\text { Decrease of TNF- } \alpha \text {, TGF- } \beta 1 \text { in both } \\
\text { groups. Fenofibrate plus pentoxifylline } \\
\text { showed significantly lower levels of } \\
\text { TNF- } \alpha \text {, direct marker linked to TGF- } \beta 1\end{array}$ \\
\hline $\begin{array}{l}\text { Faghihzadeh F } \\
{[67]}\end{array}$ & 50 & $\begin{array}{l}\text { USG, FibroScan } \\
\text { and elevated ALT }\end{array}$ & $500 \mathrm{mg}$ resveratrol & 12 weeks & $\begin{array}{l}\text { Decrease in hs-CRP, IL-6, TNF- } \alpha \text { and } \\
\text { NF } \beta \text { in resveratrol group compared with } \\
\text { the baseline and the placebo group }\end{array}$ \\
\hline Chen S [68] & 60 & USG & $\begin{array}{c}2 \text { capsules } 150 \text { mg resveratrol } \\
\text { twice daily }\end{array}$ & 3 months & Reductions in TNF- $\alpha$ \\
\hline Heebřll S [69] & 28 & Liver biopsy & $1.6 \mathrm{~g}$ resveratrol & 6 months & No change in serum TNF- $\alpha$ and CD163 \\
\hline Celinski K [71] & 74 & Liver biopsy & $\begin{array}{l}\text { Group I - Essentiale forte } \\
\quad 3 \times 1 \text { tablet/day and } \\
\text { tryptophan } 2 \times 500 \mathrm{mg} / \text { day } \\
\text { Group II }- \text { Essentiale forte } \\
\text { and melatonin } 2 \times 5 \mathrm{mg} / \text { day } \\
\text { Group III - only Essentiale }\end{array}$ & 14 months & $\begin{array}{l}\text { Lower levels of IL-1, IL- } 6 \text { and TNF- } \alpha \\
\text { only in melatonin and tryptophan, } \\
\text { compared with group III }\end{array}$ \\
\hline $\begin{array}{l}\text { Mykhal'chyshyn } \\
\text { H [73] }\end{array}$ & 72 & USG & Multiprobiotic "Symbiter" & 30 days & $\begin{array}{l}\text { Decrease in IL-6, IL-8, TNF- } \alpha \text {, IL-1 } \beta \text {, } \\
\text { IFN- } \gamma \text {. In patients with normal levels } \\
\text { of transaminases significant decrease } \\
\text { only in TNF- } \alpha \text { and IL- } 8\end{array}$ \\
\hline Quin Y [75] & 80 & USG & Fish oil (4 g/day) & 3 months & Reduced in TNF- $\alpha$ and leukotrienes B4 \\
\hline Guo H [76] & 44 & USG & $\begin{array}{l}250 \mathrm{~mL} \text { of either bayberry } \\
\text { juice or placebo twice daily }\end{array}$ & 4 weeks & Decreased in TNF- $\alpha$ and IL- 8 \\
\hline Sharifi N [77] & 56 & $\begin{array}{c}\text { USG and elevated } \\
\text { ALT }\end{array}$ & Vitamin D 50,000 IU & $\begin{array}{l}\text { Every } \\
14 \text { days for } \\
4 \text { months }\end{array}$ & No changes in serum hs-CRP \\
\hline Chen S [78] & 60 & USG & Dihydromyricetin & 3 months & Decrease in TNF- $\alpha$ \\
\hline
\end{tabular}


fylline. During 24-week observation of both therapeutic regimens in patients with NAFLD diagnosed on the basis of imaging studies or histopathological investigation, a significant beneficial effect on TNF- $\alpha$ and TGF- $\beta 1$ levels, significantly more pronounced if pentoxifylline was added to the therapy, was demonstrated [65].

Resveratrol is a phytochemical found in over 70 species of plants, including plants of economic importance such as grape (Vitis vinifera), cranberry (Vaccinium macrocarpon), and peanut (Arachis hypogaea). The mechanism of action of this compound has not been fully elucidated. It has been suggested that it inhibits cellular respiration, which is associated with its antioxidant and anti-inflammatory effect [66]. Faghihzadeh et al. demonstrated that the use of resveratrol in a daily dose of $500 \mathrm{mg}$ as a supplement to the diet and exercise for 12 weeks, compared with placebo, is associated with a significant reduction in the activity of proinflammatory cytokines (hs-CRP, IL-6, TNF- $\alpha$ and NFK $\beta$ ). It should be emphasized that in that study the signs of NAFLD were diagnosed on the basis of anamnesis, the value of elastomers and the activity of ALT, ultrasound of the liver and FibroScan, and that only 50 patients were included in the study [67]. Similar properties with respect to the effects on the immune system were demonstrated for smaller doses of the substance. The application of resveratrol in doses of two capsules 150 mg twice daily in patients with ultrasound evidence of NAFLD for a period of three months was also associated with significant reduction of TNF- $\alpha$ and fibroblast growth factor 21 (FGF-21) [68]. Conversely, Heebřll et al. found no benefit of $1.5 \mathrm{mg}$ over a 6-month period for obese patients with elevated transaminases and histopathological NAFLD/NASH. The use of this substance, compared to placebo, was not associated with an improved histological presentation of the liver. In addition, there was no significant reduction in the inflammatory markers CD163 and TNF- $\alpha$ [69].

Hepatocytes are a primary target for interleukin-22 (IL-22). It has been proven that it is associated with the production of acute phase proteins and induction of expression of proteins associated with the repair of tissues. Research results also indicate the involvement of this interleukin in protection of the liver from damage by promoting the survival and proliferation of hepatocytes. An animal model demonstrated that the use of recombinant IL-22 in the case of fatty liver is associated with protection of hepatocytes against damage and with a decrease in the concentration of TNF- $\alpha$ [70].

Based on the results of histopathological investigations confirming the presence of NAFLD signs in 56 patients, it was found that adding melatonin $(2 \times 5 \mathrm{mg} /$ day $)$ or tryptophan $(2 \times 500 \mathrm{mg} /$ day $)$ to a preparation containing phospholipids for a period of 14 months was associated with a significant reduction in IL-1, IL-6 and TNF- $\alpha$. No such effects were noted if the preparations containing phospholipids were used alone [71].

Probiotics are living microorganisms which have a number of beneficial properties, including interaction with the enterohepatic axis. It has been shown that the use of preparations containing 7 strains of these bacteria ( $\mathrm{Lac}$ tobacillus casei, Lactobacillus rhamnosus, Streptococcus thermophilus, Bifidobacterium breve, Lactobacillus acidophilus, Bifidobacterium longum, and Lactobacillus bulgaricus) and a probiotic (fructooligosaccharide) twice a day for 28 weeks in patients with NAFLD diagnosed on the basis of ALT activity and ultrasound is associated with a significant reduction of hs-CRP, TNF- $\alpha$, and TNFK-B p65 [72]. Beneficial effects of "Symbiter multiprobiotic" (Bifidobacterium, Lactobacillus, lactic streptococci, and propionic acid bacteria) have been observed in patients with NAFLD and T2DM. As demonstrated in a group of 72 patients, including 45 using multiprobiotics and hypoglycemic drugs, within 30 days of therapy the addition of probiotics is associated with a significant reduction in the levels of IL- 6 , IL- 8 , TNF- $\alpha$, IL- $1 \beta$ and IFN- $\gamma$. It is noteworthy that in patients with NAFLD and normal hepatic transaminase activity, a negative correlation was found only in the case of TNF- $\alpha$ and IL-8 [73]. Probiotics are indigestible components of nutrients that ferment in the large intestine and are responsible for the beneficial modeling of the host's intestinal flora. There are present in many vegetables such as asparagus, garlic, onion and leek. A currently ongoing study is assessing the impact of 14-week use of these compounds in a dose of $16 \mathrm{~g} /$ day on the concentration of pro-inflammatory cytokines (MCP-1, TNF- $\alpha$, IL- 6 and IL-8) in patients with BMI $\geq 25 \mathrm{~kg} / \mathrm{m}^{2}$ and diagnosed with NAFLD [74].

Qin et al., using for three months a fish oil product containing eicosapentaenoic acid (EPA) and docosahexaenoic acid (DHA) in a group of patients with NAFLD, found that it has beneficial immunomodulatory properties. The use of this preparation was associated with a significant reduction in the concentration of TNF- $\alpha$, leukotriene B4 and FGF-21. However, they did not observe a significant effect on the levels of hs-CRP. After adjustment of the study group in terms of age, sex, and BMI, they found a positive correlation between the lipid and glucose levels, GGTP activity and reduction of FGF-21 [75].

There are indications of the beneficial effects of dietary supplements in patients with NAFLD. It has been shown that the use of laurel tree juice in this group of patients significantly reduces the concentration of protein carbonyl groups $(p=0.038)$, TNF- $\alpha$ and IL-8 [76]. However, no such beneficial immunomodulatory properties were found in the case of the use of vitamin D. Its administration at a dose of 50,000 IU every 14 days for 4 months did not affect the concentration of TNF- $\alpha$ and TGF- $\beta 1$. On the other hand, a significant reduction of hs-CRP in the group using this vitamin was reported [77]. 
A beneficial effect on the modulation of the immune system has also been demonstrated for some plants, e.g. Ampelopsis grossedentata, which is available in China and some countries of eastern Asia. It has been shown that dihydromyricetin is responsible for its beneficial properties. This substance at a dose of $150 \mathrm{mg}$ administered twice a day has been found to significantly reduce the concentration of TNF- $\alpha$ and FGF-21 in patients with NAFLD [78].

\section{Conclusions}

The immune system, which is associated in particular with the enterohepatic axis and the presence of excess fat in the liver, is involved in both the development of NAFLD and in the process of disease progression. The presence of changes in this system may constitute an important diagnostic tool and may allow one to identify patients at risk of developing complications. It is believed that some of the tests assessing the immune system can be used in the primary diagnostic panel of this disease. There is also evidence for the possibility of using drugs and dietary supplements that by interfering with the immune system will suppress its proinflammatory potential, thus preventing the progression of NAFLD.

The authors declare no conflict of interest.

\section{References}

1. Anstee QM, McPherson S, Day CP (2011): How big a problem is nonalcoholic fatty liver disease? BMJ 343: d3897.

2. Chalasani N, Younossi Z, Lavine JE, et al. (2012): The diagnosis and management of non-alcoholic fatty liver disease: Practice guideline by the American Association for the Study of Liver Diseases, American College of Gastroenterology, and the American Gastroenterological Association. Am J Gastroenterol 107: 811-826.

3. Kneeman JM, Misdraji J, Corey KE (2012): Secondary causes of nonalcoholic fatty liver disease. Therap Adv Gastroenterol 5: 199-207.

4. Aizawa Y, Hokari A (2017): Autoimmune hepatitis: current challenges and future prospects. Clin Exp Gastroenterol 10: 9-18.

5. Petäjä EM, Yki-Järvinen H (2016): Definitions of normal liver fat and the association of insulin sensitivity with acquired and genetic NAFLD - a systematic review. Int J Mol Sci 7: pii:E633.

6. Neuschwander-Tetri BA (2010): Hepatic lipotoxicity and the pathogenesis of nonalcoholic steatohepatitis: The central role of nontriglyceride fatty acid metabolites. Hepatology 52: 774788.

7. Wong VW, Wong GL, Choi PC, et al. (2010): Disease progression of nonalcoholic fatty liver disease: a prospective study with paired liver biopsies at 3 years. Gut 59: 969-974.

8. Onnerhag K, Nilsson PM, Lindgren S (2014): Increased risk of cirrhosis and hepatocellular cancer during long-term follow-up of patients with biopsy-proven NAFLD. Scand J Gastroenterol 49: 1111-1118.
9. Watanabe S, Hashimoto E, Ikejima K, et al. (2015): Evidence-based clinical practice guidelines for nonalcoholic fatty liver disease/nonalcoholic steatohepatitis. J Gastroenterol 50: 364-377.

10. Ong JP, Pitts A, Younossi ZM (2008): Increased overall mortality and liver-related mortality in nonalcoholic fatty liver disease. J Hepatol 49: 608-612.

11. Sherif ZA, Saeed A, Ghavimi S, et al. (2016): Global Epidemiology of Nonalcoholic Fatty Liver Disease and Perspectives on US Minority Populations. Dig Dis Sci 61: 1214-1225.

12. Kosmalski M, Kasznicki J, Drzewoski J (2013): Relationship between ultrasound features of nonalcoholic fatty liver disease and cardiometabolic risk factors in patients with newly diagnosed type 2 diabetes. Pol Arch Med Wewn 123: 436-442.

13. Masarone M, Federico A, Abenavoli L, et al. (2014): Non alcoholic fatty liver: epidemiology and natural history. Rev Recent Clin Trials 9: 126-133.

14. Almeda-Valdes P, Aguilar-Olivos N, Uribe M, et al. (2014): Common features of the metabolic syndrome and nonalcoholic fatty liver disease. Rev Recent Clin Trials 9: 148-158.

15. Yu J, Marsh S, Hu J, et al. (2016): The Pathogenesis of Nonalcoholic Fatty Liver Disease: Interplay between Diet, Gut Microbiota, and Genetic Background. Gastroenterol Res Pract 2016: 2862173.

16. Fotbolcu H, Zorlu E (2016): Nonalcoholic fatty liver disease as a multi-systemic disease. World J Gastroenterol 22: 40794090.

17. Liang S, Cheng X, Hu Y, et al. (2017): Insulin-like growth factor 1 and metabolic parameters are associated with nonalcoholic fatty liver disease in obese children and adolescents. Acta Paediatr 106: 298-303.

18. Stefan N, Häring HU, Hu FB, et al. (2016): Divergent associations of height with cardiometabolic disease and cancer: epidemiology, pathophysiology, and global implications. Lancet Diabetes Endocrinol 4: 457-467.

19. Stern C, Castera L (2017): Non-invasive diagnosis of hepatic steatosis. Hepatol Int 11: 70-78.

20. European Association for the Study of the Liver (EASL), European Association for the Study of Diabetes (EASD), European Association for the Study of Obesity (EASO) (2016): EASL-EASDEASO clinical practice guidelines for the management of nonalcoholic fatty liver disease. J Hepatol 64: 1388-1402.

21. Bravo AA, Sheth SG, Chopra S (2001): Liver biopsy. N Engl J Med 44: 495-500.

22. Kopec KL, Burns D (2011): Nonalcoholic fatty liver disease: a review of the spectrum of disease, diagnosis, and therapy. Nutr Clin Pract 26: 565-576.

23. Jacobs WH (1989): Statement on outpatient percutaneous liver biopsy. Dig Dis Sci 34: 322-323.

24. Maleki I, Rastgar A, Hosseini V, et al. (2014): High sensitive CRP and pentraxine 3 as noninvasive biomarkers of nonalcoholic fatty liver disease. Eur Rev Med Pharmacol Sci 18: 1583-1590.

25. Bhatia LS, Curzen NP, Calder PC, et al. (2012): Non-alcoholic fatty liver disease: a new and important cardiovascular risk factor? Eur Heart J 33: 1190-1200.

26. Cernea S, Cahn A, Raz I (2017): Pharmacological management of nonalcoholic fatty liver disease in type 2 diabetes. Expert Rev Clin Pharmacol 6: 1-13.

27. Rotman Y, Sanyal AJ (2017). Current and upcoming pharmacotherapy for non-alcoholic fatty liver disease. Gut 66 : 180-190. 
28. Meli R, Mattace Raso G, Calignano A (2014): Role of innate immune response in non-alcoholic Fatty liver disease: metabolic complications and therapeutic tools. Front Immunol 5: 177.

29. Narayanan S, Surette FA, Hahn YS (2016): The immune landscape in nonalcoholic steatohepatitis. Immune Netw 16: 147-158.

30. Robinson MW, Harmon C, O'Farrelly C (2016): Liver immunology and its role in inflammation and homeostasis. Cell Mol Immunol 13: 267-276.

31. Brenner C, Galluzzi L, Kepp O, et al. (2013): Decoding cell death signals in liver infammation. J Hepatol 59: 583-594.

32. Seki E, Brenner DA (2008): Toll-like receptors and adaptor molecules in liver disease: update. Hepatology 48: 322-335.

33. Ilan Y (2016): Review article: novel methods for the treatment of non-alcoholic steatohepatitis - targeting the gut immune system to decrease the systemic inflammatory response without immune suppression. Aliment Pharmacol Ther 44: 1168-1182.

34. Papanastasiou L, Fountoulakis S, Vatalas IA 2017: Adrenal disorders and non-alcoholic fatty liver disease Minerva Endocrinol 42: 151-163.

35. Stefan N, Ramsauer M, Jordan P, et al. (2014): Inhibition of $11 \beta$-HSD1 with RO5093151 for non-alcoholic fatty liver disease: a multicentre, randomised, double-blind, placebo-controlled trial. Lancet Diabetes Endocrinol 2: 406-416.

36. Massaro KS, Costa SF, Leone C, et al. (2007): Procalcitonin (PCT) and C-reactive protein (CRP) as severe systemic infection markers in febrile neutropenic adults. BMC Infect Dis 22: 137.

37. Faix JD (2013): Biomarkers of sepsis. Crit Rev Clin Lab Sci 50: $23-36$.

38. Simon L, Gauvin F, Amre DK, et al. (2004): Serum procalcitonin and C-reactive protein levels as markers of bacterial infection: a systematic review and meta-analysis. Clin Infect Dis 39: 206-217.

39. Rami D, La Bianca M, Agostinis C, et al. (2014): The first trimester gravid serum regulates procalcitonin expression in human macrophages skewing their phenotype in vitro. Mediators Inflamm 2014: 248963.

40. Kuppan G, Anjana RM, Deepa M, et al. (2012): Inflammatory markers in relation to nonalcoholic fatty liver disease in urban South Indians. Diabetes Technol Ther 14: 152-158.

41. Oruc N, Ozutemiz O, Yuce G, et al. (2009): Serum procalcitonin and CRP levels in non-alcoholic fatty liver disease: a case control study. BMC Gastroenterol 9: 16.

42. Chang CC, Wu CL, Su WW, et al. (2015): Interferon gamma-induced protein 10 is associated with insulin resistance and incident diabetes in patients with nonalcoholic fatty liver disease. Sci Rep 5: 10096.

43. Lee YJ, Lee HR, Shim JY, et al. (2010): Relationship between white blood cell count and nonalcoholic fatty liver disease. Dig Liver Dis 42: 888-894.

44. Avanzas P, Quiles J, López de Sá E, et al. (2004): Neutrophil count and infarct size in patients with acute myocardial infarction. Int J Cardiol 97: 155-156.

45. Wang X, Zhang G, Jiang X, et al. (2014): Neutrophil to lymphocyte ratio in relation to risk of all-cause mortality and cardiovascular events among patients undergoing angiography or cardiac revascularization: a meta-analysis of observational studies. Atherosclerosis 234: 206-213.

46. Sertoglu E, Uyanik M (2014): Accurate use of neutrophil/ lymphocyte ratio from the perspective of laboratory experts. Vasc Health Risk Manag 10: 13-14.
47. Kara M, Dogru T, Genc H, et al. (2015): Neutrophil-to-lymphocyte ratio is not a predictor of liver histology in patients with nonalcoholic fatty liver disease. Eur J Gastroenterol Hepatol 27: 1144-1148.

48. Abdel-Razik A, Mousa N, Shabana W, et al. (2016): A novel model using mean platelet volume and neutrophil to lymphocyte ratio as a marker of nonalcoholic steatohepatitis in NAFLD patients: multicentric study. Eur J Gastroenterol Hepatol 28: e1-9.

49. Stojsavljević S, Gomerčić Palčić M, Virović Jukić L, et al. (2014): Adipokines and proinflammatory cytokines, the key mediators in the pathogenesis of nonalcoholic fatty liver disease. World J Gastroenterol 20: 18070-18091.

50. Plomgaard P, Bouzakri K, Krogh-Madsen R, et al. (2015): Tumor necrosis factor-alpha induces skeletal muscle insulin resistance in healthy human subjects via inhibition of Akt substrate 160 phosphorylation. Diabetes 54: 2939-2945.

51. Polyzos SA, Kountouras J, Zavos Ch (2009): The multi-hit process and the antagonistic roles of tumor necrosis factor-alpha and adiponectin in non alcoholic fatty liver disease. Hippokratia 3: 127.

52. Seo YY, Cho YK, Bae JC, et al. (2013): Tumor Necrosis Factor- $\alpha$ as a Predictor for the Development of Nonalcoholic Fatty Liver Disease: A 4-Year Follow-Up Study. Endocrinol Metab (Seoul) 28: 41-45.

53. Kamari Y, Shaish A, Vax E, et al. (2011): Lack of interleukin- $1 \alpha$ or interleukin $1 \beta$ inhibits transformation of steatosis to steatohepatitis and liver fibrosis in hypercholesterolemic mice. J Hepatol 55: 1086-1094.

54. Lagathu C, Yvan-Charvet L, Bastard JP, et al. (2006): Longterm treatment with interleukin-1beta induces insulin resistance in murine and human adipocytes. Diabetologia 49: 2162-2217.

55. Isoda K, Sawada S, Ayaori M, et al. (2005): Deficiency of interleukin-1 receptor antagonist deteriorates fatty liver and cholesterol metabolism in hypercholesterolemic mice. J Biol Chem 280: 7002-7009.

56. Polyzos SA, Kountouras J, Polymerou V, et al. (2016): Vaspin, resistin, retinol-binding protein-4, interleukin- $1 \alpha$ and interleukin-6 in patients with nonalcoholic fatty liver disease. Ann Hepatol 15: 705-714.

57. Cepero-Donates Y, Lacraz G, Ghobadi F, et al. (2016): Interleukin-15-mediated inflammation promotes non-alcoholic fatty liver disease. Cytokine 82: 102-111.

58. Li Y, Li-Li Z, Qin L, et al. (2010): Plasma interleukin-18/ interleukin-18 binding protein ratio in Chinese with NAFLD. Hepatogastroenterology 57: 103-106.

59. Giles DA, Moreno-Fernandez ME, Stankiewicz TE, et al. (2016): Regulation of Inflammation by IL-17A and IL-17F Modulates Non-Alcoholic Fatty Liver Disease Pathogenesis. PLoS One 11: e0149783.

60. Tang Y, Bian Z, Zhao L, et al. (2011): Interleukin-17 exacerbates hepatic steatosis and inflammation in non-alcoholic fatty liver disease. Clin Exp Immunol 166: 281-290.

61. Wei Y, Tian Q, Zhao X, et al. (2015): Serum transforming growth factor beta 3 predicts future development of nonalcoholic fatty liver disease. Int J Clin Exp Med 8: 4545-4550.

62. Weston CJ, Shepherd EL, Claridge LC, et al. (2015): Vascular adhesion protein-1 promotes liver inflammation and drives hepatic fibrosis. J Clin Invest 125: 501-520.

63. Larson-Meyer DE, Newcomer BR, Heilbronn LK, et al. (2008): Effect of 6-month calorie restriction and exercise on 
serum and liver lipids and markers of liver function. Obesity (Silver Spring) 16: 1355-1362.

64. Derosa G, Mugellini A, Pesce RM, et al. (2015): Perindopril and barnidipine alone or combined with simvastatin on hepatic steatosis and inflammatory parameters in hypertensive patients. Eur J Pharmacol 766: 31-36.

65. El-Haggar SM, Mostafa TM (2015): Comparative clinical study between the effect of fenofibrate alone and its combination with pentoxifylline on biochemical parameters and liver stiffness in patients with non-alcoholic fatty liver disease. Hepatol Int 9: 471-479.

66. Madrigal-Perez LA, Ramos-Gomez M (2016): Resveratrol Inhibition of Cellular Respiration: New Paradigm for an Old Mechanism. Int J Mol Sci 17: 368.

67. Faghihzadeh F, Adibi P, Rafiei R, et al. (2014): Resveratrol supplementation improves inflammatory biomarkers in patients with nonalcoholic fatty liver disease. Nutr Res 34 : 837-843.

68. Chen S, Zhao X, Ran L, et al. (2015): Resveratrol improves insulin resistance, glucose and lipid metabolism in patients with non-alcoholic fatty liver disease: a randomized controlled trial. Dig Liver Dis 47: 226-232.

69. Heebřll S, Kreuzfeldt M, Hamilton-Dutoit S, et al. (2016): Placebo-controlled, randomised clinical trial: high-dose resveratrol treatment for non-alcoholic fatty liver disease. Scand J Gastroenterol 51: 456-464.

70. Yang L, Zhang Y, Wang L, et al. (2010): Amelioration of high fat diet induced liver lipogenesis and hepatic steatosis by interleukin-22. J Hepatol 53: 339-347.

71. Celinski K, Konturek PC, Slomka M, et al. (2014): Effects of treatment with melatonin and tryptophan on liver enzymes, parameters of fat metabolism and plasma levels of cytokines in patients with non-alcoholic fatty liver disease--14 months follow up. J Physiol Pharmacol 65: 75-82.

72. Eslamparast T, Poustchi H, Zamani F, et al. (2014): Synbiotic supplementation in nonalcoholic fatty liver disease: a randomized, double-blind, placebo-controlled pilot study. Am J Clin Nutr 99: 535-542.

73. Mykhal'chyshyn HP, Bodnar PM, Kobyliak NM (2013): Effect of probiotics on proinflammatory cytokines level in patients with type 2 diabetes and nonalcoholic fatty liver disease. Lik Sprava 2: 56-62.

74. Lambert JE, Parnell JA, Eksteen B, et al. (2015): Gut microbiota manipulation with prebiotics in patients with non-alcoholic fatty liver disease: a randomized controlled trial protocol. BMC Gastroenterol 15: 169.

75. Qin Y, Zhou Y, Chen SH, et al. (2015): Fish oil supplements lower serum lipids and glucose in correlation with a reduction in plasma fibroblast growth factor 21 and prostaglandin E2 in nonalcoholic fatty liver disease associated with hyperlipidemia: A randomized clinical trial. PLoS One 10: e0133496.

76. Guo H, Zhong R, Liu Y, et al. (2014): Effects of bayberry juice on inflammatory and apoptotic markers in young adults with features of non-alcoholic fatty liver disease. Nutrition 30: 198-203.

77. Sharifi N, Amani R, Hajiani E, et al. (2014): Does vitamin D improve liver enzymes, oxidative stress, and inflammatory biomarkers in adults with non-alcoholic fatty liver disease? A randomized clinical trial. Endocrine 47: 70-80.

78. Chen S, Zhao X, Wan J, et al. (2015): Dihydromyricetin improves glucose and lipid metabolism and exerts anti-inflammatory effects in nonalcoholic fatty liver disease: A randomized controlled trial. Pharmacol Res 99: 74-81. 